\title{
ON THE UNIQUENESS QUESTION FOR HAHN-BANACH EXTENSIONS FROM THE SPACE OF $\varrho^{1}$ ANALYTIC FUNCTIONS
}

\author{
EDGAR REICH ${ }^{1}$
}

\begin{abstract}
Let $\Omega$ be a region of the complex plane, $\mathscr{B}(\Omega)$ the space of analytic $\mathcal{L}^{1}$ functions over $\Omega$, and $\kappa \in E^{\infty}(\Omega)$. An evident necessary condition for the linear functional $\Lambda_{\kappa}[\varphi]=\iint_{\Omega} \kappa \varphi d x d y(\varphi \in \mathscr{B}(\Omega))$ to have a unique Hahn-Banach extension from $9 B(\Omega)$ to $L^{\prime}(\Omega)$ is that $\left\|\Lambda_{\kappa \mid G}\right\|=\|\kappa \mid G\|_{\infty}$ for every restriction $\kappa \mid G$ of $\kappa$ to a subregion $G$ of $\Omega$. An example is constructed to show that not even a considerably stronger condition is sufficient for uniqueness of the Hahn-Banach extension. Remarks on the problem of whether $|\kappa(z)|$ is necessarily constant a.e. if the Hahn-Banach extension is unique indicate that this question is still open, contrary to an assertion in the literature.
\end{abstract}

1. Introduction. If $\Omega$ is a region of the complex plane $\mathbf{C}$ we denote by $\Re(\Omega)$ the Banach space $\sigma_{B}(\Omega)=\left\{\varphi \in \mathcal{L}^{\mathrm{l}}(\Omega): \varphi(z)\right.$ is analytic in $\left.\Omega\right\}$, with norm $\|\varphi\|=$ $\iint_{\Omega}|\varphi(z)| d x d y$. For $\kappa \in \mathcal{L}^{\infty}(\Omega)$, we consider the linear functional,

$$
\Lambda_{\kappa}[\varphi]=\iint_{\Omega} \kappa(z) \varphi(z) d x d y, \quad \varphi \in \mathscr{H}(\Omega) .
$$

In the special case $\Omega=U=\{|z|<1\}$ we shall adopt the normalizations,

$$
\|\kappa\|_{\infty}=\operatorname{ess}_{z \in U}|\kappa(z)|=1, \quad\left\|\Lambda_{\kappa}\right\|=\sup _{\varphi \in \mathscr{B}(U)} \frac{\left|\iint_{U} \kappa(z) \varphi(z) d x d y\right|}{\|\varphi\|}=1 .
$$

In this case we shall write $\kappa \in \mathrm{HBU}$ if $\Lambda_{\kappa}$ has a unique Hahn-Banach extension from $\because B(U)$ to $E^{\prime}(U)$. We are interested in the question of whether or not a given function $\kappa$ satisfying (1.1) belongs to $\mathrm{HBU}$, and some related matters. Among other motivations this problem is of interest in the context of quasiconformal mappings of the disk with specified boundary values, as statements about $\Lambda_{\kappa}$ frequently have an interpretation for such mappings. For the sake of conciseness we restrict ourselves, however, purely to the function analysis problem here. In view of the normalization (1.1), $\kappa \in \mathrm{HBU}$ if and only if the conditions

$$
\iint_{U} \kappa(z) \varphi(z) d x d y=\iint_{U} \tilde{\kappa}(z) \varphi(z) d x d y \quad \text { for all } \varphi \in \mathscr{B}(U),\|\tilde{\kappa}\|_{\infty} \leqslant 1,
$$

imply that $\kappa(z)=\tilde{\kappa}(z)$ a.e. in $U$.

Our contribution here is to the question of necessary conditions for HBU. For orientation, however, let us review something of what is known in the way of sufficient conditions.

Received by the editors July $9,1982$.

1980 Mathematics Subject Classification. Primary 46E15.

${ }^{1}$ Work done with support from NSF grant MCS-810760. 
The most trivial example of an element of $\mathrm{HBU}$ is $\kappa(z)=\overline{\varphi_{0}(z)} /\left|\varphi_{0}(z)\right|$, where $\varphi_{0} \in \Re(U)$. Considerably more general sufficient conditions are known $[\mathbf{2}, \mathbf{4}, \mathbf{5}]$. We list two results of this type below.

THEOREM 1.1 [4]. Suppose $\kappa(z)=\overline{\varphi_{0}(z)} /\left|\varphi_{0}(z)\right|, z \in U$, and there exists a sequence $\varphi_{n} \in \Re(U), n=1,2,3, \ldots$, such that $\lim \varphi_{n}(z)=\varphi_{0}(z)$ a.e., and $\lim \iint_{U}\left[\left|\varphi_{n}\right|-\operatorname{Re}\left(\kappa \varphi_{n}\right)\right] d x d y=0$. Then $\kappa \in \mathrm{HBU}$.

THEOREM $1.2[2] .^{2}$ Suppose $\varphi_{0}(z)$ is analytic in $U$, and

$$
(1-r) \int_{0}^{2 \pi}\left|\varphi_{0}\left(r e^{i \theta}\right)\right| d \theta \leqslant 1, \quad 0 \leqslant r<1 .
$$

Then $\overline{\varphi_{0}(z)} /\left|\varphi_{0}(z)\right| \in \mathrm{HBU}$.

There are no known examples of $\kappa$ belonging to $\mathrm{HBU}$ for which (neglecting subsets of $U$ of measure 0$)|\kappa(z)|$ is nonconstant.

2. Necessary conditions. If $G$ is a region, $G \subset U$, we shall say that condition (R) holds for $G$ if

$$
\sup _{\varphi \in \mathscr{B}(G)} \frac{\left|\iint_{G} \kappa(z) \varphi(z) d x d y\right|}{\iint_{G}|\varphi(z)| d x d y}=\underset{z \in G}{\operatorname{ess} \sup }|\kappa(z)| .
$$

On the other hand, if $E \subset U$, where $E$ is an arbitrary set of positive measure, we shall say that condition (S) holds for $E$ if

$$
\sup _{\varphi \in \mathscr{B}(U)} \frac{\left|\iint_{E} \kappa(z) \varphi(z) d x d y\right|}{\iint_{E}|\varphi(z)| d x d y}=\underset{z \in E}{\operatorname{ess} \sup }|\kappa(z)|=1
$$

The conclusion that ( $\mathrm{R})$ holds for all regions $G, G \subset U$, is an elementary consequence of the hypothesis $\kappa \in \mathrm{HBU}$, as has been observed by Šretov [6]. Since the restriction to $G$ of members of $\mathscr{B}(U)$ belongs to $\mathscr{B}(G)$, and since we are assuming (1.1), a stronger necessary condition actually holds; namely,

THEOREM 2.1. $\kappa \in \mathrm{HBU} \Rightarrow(\mathrm{S})$ holds for all $E \subset U$ such that Int $E \neq \varnothing \Rightarrow(\mathrm{R})$ holds for all $G \subset U$.

Proof. This is also completely elementary. Namely, let $\Re(U)$ denote the null space, $\mathscr{T}(U)=\left\{\nu \in \mathscr{L}^{\infty}(U): \Lambda_{\nu}[\varphi]=0\right.$ for all $\left.\varphi \in \mathscr{B}(U)\right\}$. If $D=\left\{\left|z-z_{0}\right|<h\right\}$ $\subset U$, and if

$$
\nu_{D}(z)= \begin{cases}\left(z-z_{0}\right) / h, & \left|z-z_{0}\right|<h, \\ 0, & \left|z-z_{0}\right|>h,\end{cases}
$$

then $\left|\nu_{D}(z)\right| \leqslant 1$, and, by Cauchy's theorem, $\nu_{D} \in \Re(U)$.

Suppose, for some $E \subset U$, and $D \subset E$,

$$
\sup _{\varphi \in \mathscr{B}(U)} \frac{\left|\iint_{E} \kappa(z) \varphi(z) d x d y\right|}{\iint_{E}|\varphi(z)| d x d y}=a<1 .
$$

\footnotetext{
${ }^{2}$ This result is stated in [2] in the context of extremal quasiconformal mappings. However, in view of the fact that it is derived as a corollary of Theorem B of [5] the formulation used above follows by Theorem $\mathrm{C}$ of [5].
} 


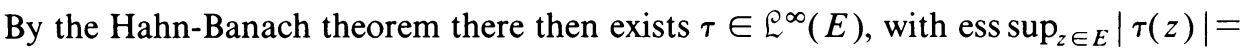
$a$, such that

$$
\iint_{E} \kappa(z) \varphi(z) d x d y=\iint_{E} \tau(z) \varphi(z) d x d y, \text { for all } \varphi \in \mathscr{B}(U) .
$$

Let

$$
\tilde{\kappa}(z)= \begin{cases}\kappa(z), & z \in U \backslash E, \\ \tau(z)+(1-a) \nu_{D}(z) / 2, & z \in E .\end{cases}
$$

Then $\|\tilde{\kappa}\|_{\infty} \leqslant 1$, and (1.2) would hold, even though $\kappa$ and $\tilde{\kappa}$ differed on the set $D$.

We will now show, by example, that even the stronger of the necessary conditions of Theorem 2.1 is not sufficient. Actually, our result will be stronger still. We shall say that condition (S)* holds if (S) holds for all $E \subset U,|E|>0$.

THEOREM 2.2. There exists $\kappa \in \mathcal{L}^{\infty}(U)$ such that $(\mathrm{S})^{*}$ holds, but such that $\kappa \notin \mathrm{HBU}$.

ThE EXAMPLE. Let $\zeta=\Phi_{0}(z)$ map $U$ conformally onto the parabolic region

$$
\Omega_{0}=\left\{\zeta=\xi+i \eta: \eta^{2}<\xi, \xi>0\right\} \text {. }
$$

We define $\kappa(z)$ as

$$
\kappa(z)=\frac{\overline{\Phi_{0}^{\prime}(z)}}{\Phi_{0}^{\prime}(z)}, \quad z \in U .
$$

If $f(\zeta)=\varphi(z)\left[\Phi_{0}^{\prime}(z)\right]^{-2}\left(z=\Phi_{0}^{-1}(\zeta) \in U\right)$, then $\varphi \in \Re(U)$ if and only if $f \in \Re\left(\Omega_{0}\right)$. Expressed in terms of $\zeta$, our objectives are then, firstly, to show that

$$
\sup _{f \in \mathscr{B}\left(\Omega_{0}\right)} \frac{\left|\iint_{E} f(\zeta) d \xi d \eta\right|}{\iint_{E}|f(\zeta)| d \xi d \eta}=1 \quad \text { for each } E \subset \Omega,|E|>0 .
$$

Secondly, we shall find a function $\tau(\zeta), \zeta \in \Omega_{0}$, with

$$
|\tau(\zeta)| \leqslant 1, \quad\|\tau-1\|_{\infty}>0
$$

such that

$$
\iint_{\Omega_{0}} f(\zeta) d \xi d \eta=\iint_{\Omega_{0}} \tau(\zeta) f(\zeta) d \xi d \eta \quad \text { for all } f \in \mathscr{B}\left(\Omega_{0}\right)
$$

To obtain $\tilde{\kappa}(z), z \in U$, satisfying (1.2), one then defines

$$
\tilde{\kappa}(z)=\tau(\zeta) \overline{\Phi_{0}^{\prime}(z)} / \Phi_{0}^{\prime}(z), \quad z=\Phi_{0}^{-1}(\zeta), \zeta \in \Omega_{0} .
$$

For a given set $E \subset \Omega,|E|>0$, let $l(\xi)=\int_{\xi+i \eta \in E} d \eta,(\xi>0)$, and, for $s$ real and positive, let $L(s)$ be the Laplace transform $L(s)=\int_{0}^{\infty} e^{-s \xi} l(\xi) d \xi$. Since $l(\xi) \leqslant 2 \xi^{1 / 2}$, we have

$$
L(s)=O\left(s^{-3 / 2}\right), \quad s \searrow 0 .
$$

Also, $L^{\prime}(s)=-\int_{0}^{\infty} \xi e^{-s \xi} l(\xi) d \xi, s>0$. We claim that

$$
\frac{\lim }{s \searrow 0} \frac{-s^{2} L^{\prime}(s)}{L(s)}=0 .
$$


For, otherwise, there would exist $\delta>0$, and $s_{0}>0$, such that

$$
L^{\prime}(s)+\delta s^{-2} L(s) \leqslant 0, \quad 0<s \leqslant s_{0},
$$

and hence $e^{-\delta / s} L(s)$ would be positive and decreasing for $0<s \leqslant s_{0}$, contradicting (2.5). Choose $\left\{s_{n}\right\}, 0<s_{n+1}<s_{n}, n=1,2,3, \ldots, \lim s_{n}=0$, such that

$$
\lim _{n \rightarrow \infty} \frac{s_{n}^{2} L^{\prime}\left(s_{n}\right)}{L\left(s_{n}\right)}=0 \text {, }
$$

and set

$$
f_{n}(\zeta)=e^{-s_{n} \zeta}, \quad n=1,2,3, \ldots, \zeta \in \Omega_{0} .
$$

Evidently, $f_{n} \in \mathscr{B}\left(\Omega_{0}\right), n=1,2,3, \ldots$, and, by $(2.6)$,

$$
\lim _{n \rightarrow \infty} \frac{s_{n}^{2} \int_{0}^{\infty} \xi e^{-s_{n} \xi} l(\xi) d \xi}{J_{n}(E)}=0,
$$

where $J_{n}(E)=\int_{0}^{\infty} e^{-s_{n} \xi} l(\xi) d \xi=\iint_{E}\left|f_{n}(\zeta)\right| d \xi d \eta$.

Let $I_{n}(E)=\iint_{E} f_{n}(\zeta) d \xi d \eta$. We have

$$
\begin{aligned}
J_{n}-\left|I_{n}\right| & \leqslant J_{n}-\operatorname{Re} I_{n}=\iint_{E} e^{-s_{n} \xi}\left[1-\cos \left(s_{n} \eta\right)\right] d \xi d \eta=2 \iint_{E} e^{-s_{n} \xi} \sin ^{2} \frac{s_{n} \eta}{2} d \xi d \eta \\
& \leqslant \frac{s_{n}^{2}}{2} \iint_{E} \eta^{2} e^{-s_{n} \xi} d \xi d \eta \leqslant \frac{s_{n}^{2}}{2} \iint_{E} \xi e^{-s_{n} \xi} d \xi d \eta \\
& =\frac{s_{n}^{2}}{2} \int_{0}^{\infty} \xi e^{-s_{n} \xi} l(\xi) d \xi .
\end{aligned}
$$

In view of (2.7), it follows that $\lim _{n \rightarrow \infty}\left|I_{n}(E)\right| / J_{n}(E)=1$. This proves (2.2).

The existence of a function $\tau(\zeta)$ satisfying (2.3) and (2.4) is known [3, Case $\alpha=2$ ]. We present below the simplified explicit formula (2.9) and a direct verification.

Let $H(\zeta)=\left(\xi-\eta^{2}\right) /(1+\xi), \zeta=\xi+i \eta \in \Omega_{0}$. We have

$$
0 \leqslant H(\zeta) \leqslant \frac{\xi}{1+\xi}<1, \quad \zeta \in \Omega_{0}
$$

and

$$
H_{\bar{\zeta}}=\frac{1+\eta^{2}}{2(1+\xi)^{2}}-\frac{i \eta}{1+\xi} .
$$

Defining

$$
\tau(\zeta)=1-H_{\xi}=1-\frac{1+\eta^{2}}{2(1+\xi)^{2}}+i \frac{\eta}{1+\xi}
$$

we obtain

$$
\begin{aligned}
|\tau(\zeta)|^{2} & =1-\frac{1}{(1+\xi)^{2}}+\frac{\left(1+\eta^{2}\right)^{2}}{4(1+\xi)^{4}} \leqslant 1-\frac{1}{(1+\xi)^{2}}+\frac{(1+\xi)^{2}}{4(1+\xi)^{4}} \\
& =1-\frac{3}{4(1+\xi)^{2}}, \quad \zeta \in \Omega_{0},
\end{aligned}
$$

so that (2.3) holds. 
To verify (2.4) one can restrict oneself to functions $f \in \mathcal{G}_{(}\left(\Omega_{0}\right)$ that are analytic on the parabola $\partial \Omega_{0}$. This is due to the fact that $\Phi_{0}^{-1}(\zeta)$ is analytic on $\partial \Omega_{0}$, and the fact that functions analytic for $|z| \leqslant 1$ are obviously dense in $\mathscr{B}(U)$. For a given $f \in \Re\left(\Omega_{0}\right)$ there exists a sequence $\xi_{n}>\infty$ such that

$$
\lim _{n \rightarrow \infty} \int_{\xi_{n}+i \eta \in \Omega_{0}}\left|f\left(\xi_{n}+i \eta\right)\right| d \eta=0 .
$$

Let $\Omega_{n}=\left\{\zeta \in \Omega_{0}: \operatorname{Re} \zeta<\xi_{n}\right\}, n=1,2,3, \ldots$ Then, since $H=0$ on $\partial \Omega_{0}$,

$$
\iint_{\Omega_{n}} H_{\zeta} f d \xi d \eta=\frac{1}{2 i} \int_{\partial \Omega_{n}} H f d \zeta=\frac{1}{2} \int_{\operatorname{Re} \zeta=\xi_{n}} H f d \eta
$$

By (2.8), (2.10), we obtain

$$
\iint_{\Omega_{0}} H_{\zeta} f d \xi d \eta=\lim \iint_{\Omega_{n}} H_{\zeta}^{-} f d \xi d \eta=0
$$

so that (2.4) follows.

3. An open question. If $\kappa \in \mathrm{HBU}$ then it follows by Theorem 2.1, that

$$
\underset{z \in E}{\operatorname{ess} \sup }|\kappa(z)|=1 \quad \text { for each open set } E, E \subset U .
$$

A natural question is whether (HBU) actually implies that

$$
|\kappa(z)|=1 \text { a.e. }
$$

Condition (3.2) would certainly hold for $\kappa \in \mathrm{HBU}$ if HBU could be shown to imply $(\mathrm{S})^{*}$. We are faced, however, by the following fact which prevents the proof of Theorem 2.1 to extend to the stronger conclusion.

Lemma 3.1. There exist sets $E \subset U,|E|>0$, with the property that $\nu \in \Re(U)$, supp $\nu \subset E \Rightarrow \nu=0$.

Proof. ${ }^{3}$ The assertion holds for any compact nowhere dense set $E$ of positive measure, that does not disconnect $U$. In fact, if $\nu \in \mathcal{K}(U)$, then

$$
F(\zeta)=-\frac{1}{\pi} \iint_{E} \frac{\nu(z)}{z-\zeta} d x d y=0, \quad|\zeta| \geqslant 1 .
$$

Since $F$ is analytic in $\mathrm{C} \backslash E$, we therefore have $F(\zeta)=0, \zeta \in U \backslash E$. But $F$ is continuous in $U$. Hence, $F(\zeta)=0, \zeta \in U$. It follows that $\nu(z)=F_{\bar{z}}=0$ a.e.

In [6] an assertion is made [6, Theorem 3, last part] which, if correct, would be tantamount to the assertion that (HBU) implies (3.2). However the reasoning in [6] overlooks the possibility that the set $E=\{z \in U:|\kappa(z)|<1\}$ could be of the foregoing type; so, whether or not (3.2) actually follows from HBU must still be considered open.

\section{REFERENCES}

1. Lennart Carleson, Mergelyan's Theorem on uniform polynomial approximation, Math. Scand. 15 (1964), 167-175.

2. W. K. Hayman and Edgar Reich, On Teichmüller mappings of the disk, Complex Variables 1 (1982) (to appear).

\footnotetext{
${ }^{3}$ This is just Lavrentev's part of Mergelyan's Theorem proved by Carleson's method [1].
} 
3. Edgar Reich, Uniqueness of Hahn-Banach extensions from certain spaces of analytic functions, Math. Z. 167 (1979), 81-89.

4. __ A criterion for unique extremality of Teichmüller mappings, Indiana Univ. Math. J. 30 (1981), 441-447.

5. On criteria for unique extremality of Teichmüller mappings, Ann. Acad. Sci. Fenn. A.I. Math. 6 (1981), 289-302.

6. V. G. Šeretor. Lrsallı extremal quasiconformal mappings, Dokl. Akad. Nauk SSSR 250 (1980), 1338-1340 = Soviet Math. Dokl. 21 (1980), 343-345.

School of Mathematics. University of Minnesota, Minneapolis, Minnesota 55455 